\title{
Anastomosing haemangioma of liver: A case report
}

\author{
XIUHUA PENG, JIN LI and ZONGHUI LIANG \\ Department of Radiology, Shanghai Jing'an District Centre Hospital, Shanghai 200040, P.R. China
}

Received May 29, 2016; Accepted July 22, 2017

DOI: $10.3892 /$ mco.2017.1341

\begin{abstract}
Anastomosing haemangioma ( $\mathrm{AH})$ is a recently described, unusual variant of capillary hemangioma that appears to be unique to the genitourinary system, with a particular proclivity for the kidney. AH is a subtype of capillary haemangioma, which is rarely encountered in clinical practice, particularly in the liver. We herein present the case of a 57-year-old woman with an incidental finding on magnetic resonance imaging of a local lesion in the liver, sized $3.3 \times 3.0 \mathrm{~cm}$. The patient underwent hepatectomy with a good postoperative recovery. The histopathological diagnosis was $\mathrm{AH}$ of the liver. To the best of our knowledge, this is the first case report of hepatic AH.
\end{abstract}

\section{Introduction}

Anastomosing haemangioma (AH) is a subtype of capillary haemangioma that is rarely encountered in clinical practice, particularly in the liver. $\mathrm{AH}$ is a recently described and unusual variant of capillary hemangioma that appears to be unique to the genitourinary system, with a particular proclivity for the kidney. Previous studies provide strong evidence supporting the benign behavior of $\mathrm{AH}$, whereas others suggest that less aggressive treatment may be preferable. Thus, the minimally invasive strategy, which is diagnostic biopsy and no treatment, should be weighed against the potential of significant tissue damage due to the growing lesion over time $(1,2)$. We herein present a rare case of pathologically confirmed $\mathrm{AH}$ in the liver in a 57-year-old female patient.

\section{Case report}

A 57-year-old Chinese woman with no significant medical history was incidentally found to have a hepatic lesion on ultrasound imaging during a routine health check-up on September 20, 2014. A mass was identified on color Doppler

Correspondence to: Dr Zonghui Liang, Department of Radiology, Shanghai Jing'an District Centre Hospital, 259 Xikang Road, Jing'an, Shanghai 200040, P.R. China

E-mail: liangzh@vip.163.com

Key words: anastomosing haemangioma, magnetic resonance imaging, hepatic imaging, sized $\sim 3.3 \times 2.8 \mathrm{~cm}$. The possibility of malignancy was high, and metastasis was highly unlikely. The lesion was further evaluated with an abdominal contrast-enhanced magnetic resonance imaging (MRI) scan. T1-weighted images (WI) on MRI revealed a round, well circumscribed, homogeneously hypointense mass, sized $\sim 3.3 \times 3.0 \mathrm{~cm}$, in the right lobe of the liver (Fig. 1A), which was hyperintense on T2WI (Fig. 1B and D) and diffusion WI (DWI; Fig. 1C). The lesion exhibited a strong contrast enhancement in the periphery during the early phase (Fig. 1E) and homogeneously persistent enhancement with a well-circumscribed boundary during the late phase (Fig. 1F). A positron emission tomography-computed tomography (PET-CT) scan revealed a low-density mass in the right hepatic lobe, with lower glycometabolism compared with the normal liver. The patient consented to receiving a hepatectomy in December 5, 2014, and she remained alive and disease-free during the 12-month postoperative follow-up (last follow-up, December 30, 2015). The patient consented to the publication of the case details.

The tumour was located in the VIII segment of the liver in close proximity to the right hepatic vein. There were no lymph node metastases and no tumour thrombi were identified in the liver. On macroscopic inspection, the resected specimen was a well-circumscribed, homogeneous, encapsulated nodular mass, sized $3.5 \times 3 \times 3 \mathrm{~cm}$ and the cut surface of the tumour was fleshy and red-gray. On microscopic examination, various numbers of blood cells were observed in the anastomosing vessels and differently sized vascular compartments. The immunohistochemical staining for CD31 was positive. The histopathological appearance together with the immunophenotypic characteristics of this tumour were indicative of $\mathrm{AH}$ of the liver.

\section{Discussion}

Hemangiomas are more commonly located in the skin and subcutaneous tissues. Visceral hemangiomas are generally not that common and occur mostly in the liver. Histologically, hemangiomas have been broadly classified as cavernous and capillary $(3,4)$. Hepatic hemangioma $(\mathrm{HH})$ is the most common benign tumour of the liver during infancy (5). On the basis of its distribution in the liver, hemangiomas may be classified as focal, multifocal, or diffuse $(6,7)$. The majority of hepatic hemangiomas are of the cavernous type, followed by the capillary type. $\mathrm{AH}$ is a rare subtype of hemangioma. AHs are distinguished from their cavernous counterparts by the anastomosing sinusoidal-like pattern of tightly packed 

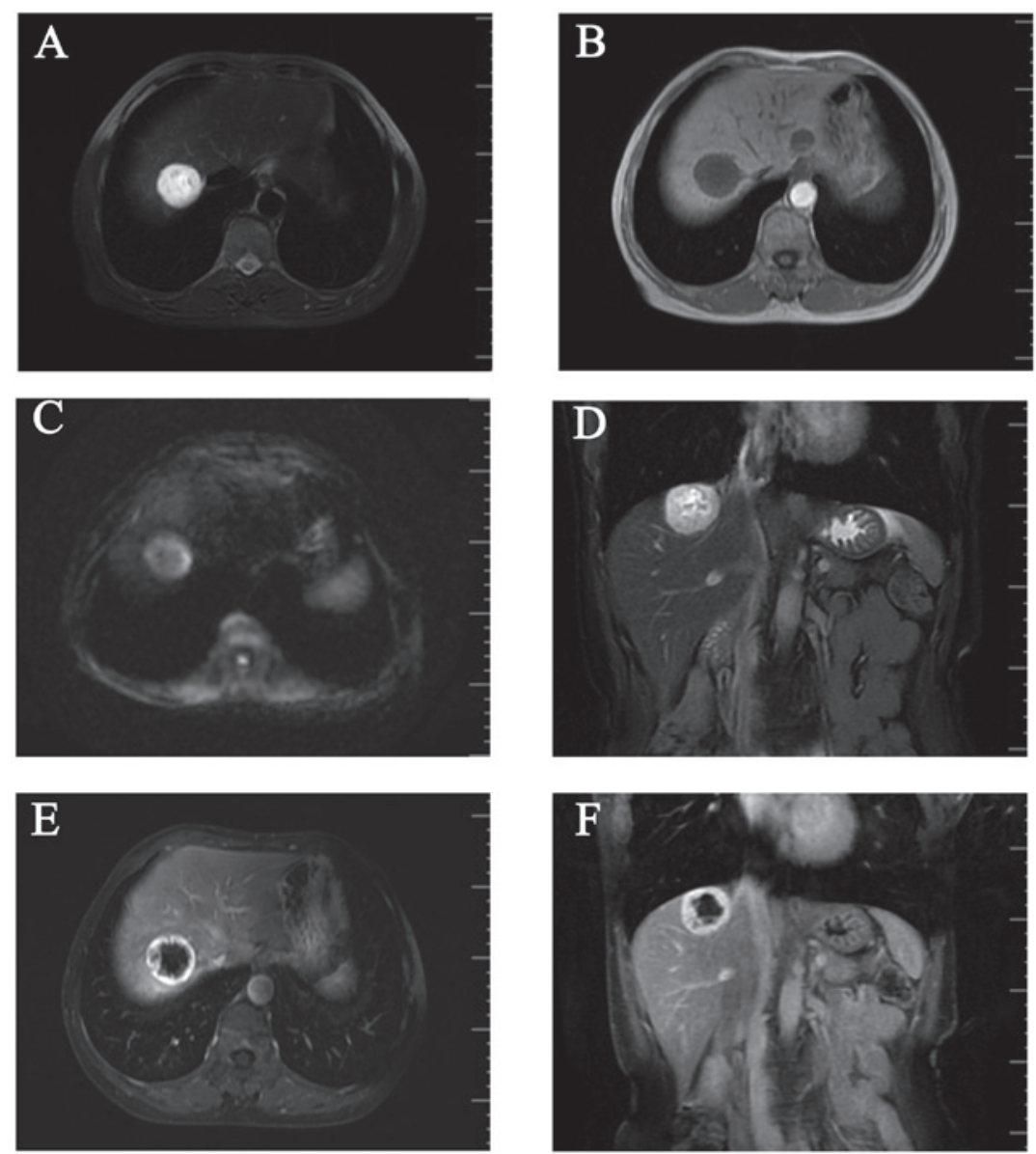

Figure 1. Contrast-enhanced magnetic resonance imaging scan. A round, well-circumscribed mass sized $\sim 3.3 \times 3.0 \mathrm{~cm}$ was identified in the right hepatic lobe, (A) homogeneously hypointense on T1-weighted images (WI), exhibiting (B) marked hyperintensity on T2WI and (C) slight hyperintensity on diffusion WI. (D) The mass exhibited hyperintensity on coronal T2WI, with (E) strong contrast enhancement in the periphery during the early phase and (F) persistent enhancement with a well-circumscribed boundary during the late phase.

capillary channels. Montgomery and Epstein described 6 cases of new variants of benign vascular tumours involving the kidneys, perinephric adipose tissue and testes, which the authors classified as AHs in 2009 (6). Subsequently, more cases of this vascular tumour have been reported in the adrenal gland (7), breast (8), liver and gastrointestinal tract (9). The mean size of this tumour in the liver is reportedly $\sim 3.35 \mathrm{~cm}$, which is larger compared with that in other organs (10). The incidence in male patients suggests a slight male predilection, with a reported male:female ratio of 1.8:1 (3). The association between end-stage renal disease and malignant renal epithelial neoplasms is well-documented (11).

Radiologically, as the number of reported cases of $\mathrm{AH}$ of the liver is limited, imaging information on the characteristics of hepatic AHs is also limited. Tao et al reported that the unenhanced axial CT scan showed a mass with a round, well-circumscribed outline, appearing to be heterogeneous. The boundary of the lesion exhibited an obviously annular and nodular enhancement in the arterial phase of the contrast-enhanced CT scan. In the venous phase, the lesion exhibited further intense enhancement. The lesion displayed homogeneously persistent enhancement and a well-circumscribed boundary on delayed images (10). A contrast-enhanced $\mathrm{CT}$ of the abdomen and pelvis confirmed a suspicious $3.4-\mathrm{cm}$ renal mass, located between the left and middle part of the liver. A contrast-enhanced MRI scan revealed a round, well-demarcated mass in the upper pole of the right kidney, homogeneously hyperintense, measuring $2.3 \times 2.1 \mathrm{~cm}$ (12).

In the present case, a contrast-enhanced abdominal MRI scan revealed a round, well-circumscribed mass sized $\sim 3.3 \times 3.0 \mathrm{~cm}$ in the right hepatic lobe, homogeneously hypointense on T1WI and hyperintense on T2WI and DWI. The lesion exhibited a strong contrast enhancement in the periphery during the early phase, with homogeneously persistent enhancement and a well-circumscribed boundary during the late phase.

Macroscopically, AHs are reportedly $0.1-6 \mathrm{~cm}$ in diameter and are well-demarcated, always unencapsulated, with a mahogany brown spongy appearance, without grossly evident necrosis or vascular invasion $(3,4,8,9,13,14)$. On pathological examination, the characteristic cytoarchitectural features of $\mathrm{AH}$ include numerous thin-walled vascular channels exhibiting a complex anastomosing growth pattern, but no evident endothelial atypia or multilayering (14). Immunohistochemical studies demonstrated that the tumour cells were diffusely positive for CD34 and CD31, the stromal cells were positive for smooth muscle actin, and the Ki-67 indicates a low proliferative activity of the tumour cells (15). In the present case, the tumour was macroscopically fleshy and red-gray. The tumour cells were abundant and formed anastomosing vascular 
channels. The immunohistochemical staining for CD31 was positive.

The differential diagnosis of $\mathrm{AH}$ includes hepatocellular carcinoma (HCC), primary hepatic angiosarcoma (PHA) and hepatic angiomyolipoma (AML). Generally, HCC patients often have a history of chronic liver disease or cirrhosis and high serum $\alpha$-fetoprotein (AFP) levels. The lesion exhibited strong contrast enhancement in the early phase and weakened enhancement in the late phase. Angiosarcoma, a subtype of soft tissue sarcoma, is an aggressive malignant disease derived from the endothelium of lymphatics or blood vessels (16). The typical imaging manifestation of PHA is moderate to marked enhancement at the peripheral and central area of the tumour during the arterial phase, which persists during the portal and delayed phases; in addition, in the majority of the cases, the central area of the lesion cannot be completely filled with the contrast agent. PHA is often accompanied by bleeding and is enclosed in a capsule. The majority of the PHA cases are accompanied by obvious clinical symptoms and compromised liver function. AML is a unique mesenchymal neoplasm consisting of blood vessels, smooth muscle and adipose cells (17). When the liver lesions exhibit early enhancement, peripheral enhancement, no capsule, the AFP levels are normal and there is no cirrhosis, the diagnosis of hepatic $\mathrm{AH}$ should be considered (18).

\section{References}

1. Brown JG, Folpe AL, Rao P, Lazar AJ, Paner GP, Gupta R, Parakh R, Cheville JC and Amin MB: Primary vascular tumors and tumor-like lesions of the kidney: A clinicopathologic analysis of 25 cases. Am J Surg Pathol 34: 942-949, 2010.

2. Wetherell DR, Skene A, Manya K, Manecksha RP, Chan Y and Bolton DM: Anastomosing haemangioma of the kidney: A rare morphological variant of haemangioma characteristic of genitourinary tract location. Pathology 45: 193-196, 2013.

3. Meyers RL: Tumors of the liver in children. Surg Oncol 16: 195-203, 2007.

4. Christison-Lagay ER, Burrows PE, Alomari A, Dubois J, Kozakewich HP, Lane TS, Paltiel HJ, Klement G, Mulliken JB and Fishman SJ: Hepatic hemangiomas: Subtype classification and development of a clinical practice algorithm and registry. J Pediatr Surg 42: 62-68, 2007.

5. Kulungowski AM, Alomari AI, Chawla A, Christison-Lagay ER and Fishman SJ: Lessons from a liver hemangioma registry: Subtype classification. J Pediatr Surg 47: 165-170, 2012.

6. Montgomery E and Epstein JI: Anastomosing hemangioma of the genitourinary tract: A lesion mimicking angiosarcoma. Am J Surg Pathol 33: 1364-1369, 2009.

7. Ross M, Polcari A, Picken M, Sankary H and Milner J: Anastomosing hemangioma arising from the adrenal gland. Urology 80: e27-e28, 2012.
8. Brehm B, Rauh C, Dankerl P and Schulz-Wendtland R: Anastomosing hemangioma in the male breast-a rarity). Rofo 186: 80-81, 2014.

9. Lin J, Bigge J, Ulbright TM and Montgomery E: Anastomosing hemangioma of the liver and gastrointestinal tract: An unusual variant histologically mimicking angiosarcoma. Am J Surg Pathol 37: 1761-1765, 2013.

10. Tao LL, Dai Y, Yin W and Chen J: A case report of a renal anastomosing hemangioma and a literature review: An unusual variant histologically mimicking angiosarcoma. Diagn Pathol 9: 159, 2014.

11. Kryvenko ON, Haley SL, Smith SC, Shen SS, Paluru S, Gupta NS, Jorda M, Epstein JI, Amin MB and Truong LD: Haemangiomas in kidneys with end-stage renal disease: A novel clinicopathological association. Histopathology 65: 309-318, 2014.

12. Zhao M, Li C, Zheng J and Sun K: Anastomosing hemangioma of the kidney: A case report of a rare subtype of hemangioma mimicking angiosarcoma and review of the literature. Int J Clin Exp Pathol 6: 757-765, 2013.

13. Kryvenko ON, Gupta NS, Meier FA, Lee MW and Epstein JI: Anastomosing hemangioma of the genitourinary system: Eight cases in the kidney and ovary with immunohistochemical and ultrastructural analysis. Am J Clin Pathol 136: 450-457, 2011.

14. Mehta V, Ananthanarayanan V, Antic T, Krausz T, Milner J, Venkataraman G and Picken MM: Primary benign vascular tumors and tumorlike lesions of the kidney: A clinicopathologic analysis of 15 cases. Virchows Arch 461: 669-676, 2012.

15. Omiyale AO: Anastomosing hemangioma of the kidney: A literature review of a rare morphological variant of hemangioma. Ann Transl Med 3: 151, 2015.

16. Heidegger I, Pichler R, Schäfer G, Zelger B, Zelger B, Aigner F, Bektic $\mathbf{J}$ and Horninger W: Long-term follow up of renal anastomosing hemangioma mimicking renal angiosarcoma. Int $\mathrm{J}$ Urol 21: 836-838, 2014.

17. Cai PQ, Wu YP, Xie CM, Zhang WD, Han R and Wu PH: Hepatic angiomyolipoma: CT and MR imaging findings with clinical-pathologic comparison. Abdom Imaging 38: 482-489, 2013.

18. Li T, Wang L, Yu HH, Sun HC, Qin LX, Ye QH, Fan J and Tang ZY: Hepatic angiomyolipoma: A retrospective study of 25 cases. Surg Today 38: 529-535, 2008. 